\title{
WELL-BOUNDED AND SCALAR-TYPE SPECTRAL OPERATORS ON SPACES NOT CONTAINING $c_{0}$
}

\author{
IAN DOUST
}

(Communicated by Paul S. Muhly)

\begin{abstract}
It is known that a necessary and sufficient condition for a wellbounded operator on a weakly complete complex Banach space to be scalar-type spectral is that its decomposition of the identity be of bounded variation. We show in this paper that this condition is necessary and sufficient exactly when the Banach space does not contain a subspace isomorphic to $c_{0}$.
\end{abstract}

A well-bounded operator on a Banach space $X$ is one which admits a functional calculus for the absolutely continuous functions on some compact interval of the real line. It is often of interest to know whether a well-bounded operator is scalar-type spectral. Scalar-type spectral operators possess a larger functional calculus and give rise to unconditional, rather than conditional, spectral expansions. Whereas scalar-type spectral operators can be represented as an integral with respect to a spectral measure, a well-bounded operator on $X$ possesses a representation in terms of a uniformly bounded increasing family of projections known as a decomposition of the identity for $X$. This representation is much less satisfactory than that for scalar-type spectral operators since if $T$ is a well-bounded operator on $X$, the projections in an associated decomposition of the identity act not on $X$ but on $X^{*}$. Furthermore, the decomposition of the identity is not in general uniquely determined by the well-bounded operator. If the operator $T \in B(X)$ possesses a functional calculus for the absolutely continuous functions on the compact interval $[a, b] \subset \mathbf{R}$, then $T$ and any decomposition of the identity $\{E(\lambda)\}_{\lambda \in \mathbf{R}}$ associated with $T$ are related by the condition that

$$
\left\langle T x, x^{*}\right\rangle=b\left\langle x, x^{*}\right\rangle-\int_{a}^{b}\left\langle x, E(\lambda) x^{*}\right\rangle d \lambda
$$

for all $x \in X$ and $x^{*} \in X^{*}$. We refer the reader who is not familiar with the above notions to [2] where the theories of spectral and well-bounded operators are fully developed.

Received by the editors April 14, 1988.

1980 Mathematics Subject Classification (1985 Revision). Primary 47B40; Secondary 46B25.

Key words and phrases. Well-bounded operators, scalar-type spectral operators.

The author acknowledges receipt of an Edinburgh University Postgraduate Studentship and an ORS award whilst this research was undertaken. 
In [1], Berkson and Dowson found a condition on the decompositions of the identity associated with a well-bounded operator $T$ which is sufficient to allow one to construct a spectral measure.

Definition. A decomposition of the identity for $X\{E(\lambda)\}_{\lambda \in \mathbf{R}}$ is said to be of bounded variation if the function $\lambda \mapsto\left\langle x, E(\lambda) x^{*}\right\rangle$ is of bounded variation for all $x \in X$ and all $x^{*} \in X^{*}$.

Theorem 1 [1, Theorem 5.2]. Suppose that $T$ is a bounded linear operator on a complex Banach space $X$ and that $\sigma(T) \subset \mathbf{R}$. Then the following are equivalent:

(i) $T$ is well-bounded and possesses a decomposition of the identity of bounded variation;

(ii) there exists a compact interval $[a, b] \subset \mathbb{R}$ and a constant $M$ such that

$$
\|g(T)\| \leq M \sup _{[a, b]}|g|
$$

for all polynomials $g$;

(iii) $T^{*}$ is a scalar-type prespectral operator of class $X$.

In general it is possible for $T$ to possess a decomposition of the identity of bounded variation without $T$ being scalar-type spectral. However, using the following two facts, Berkson and Dowson showed that this cannot happen if $X$ is weakly complete. On such spaces, every well-bounded operator whose decomposition of the identity is of bounded variation is real scalar-type spectral (i.e., is a scalar-type spectral operator whose spectrum is contains in $\mathbf{R}$ ).

Fact 1 [2, Theorem 6.24]. An operator $T \in B(X)$ is scalar-type spectral if and only if it has a weakly compact $\mathscr{C}(\sigma(T))$ functional calculus (i.e., for all $x \in X$, the map $f \mapsto f(T) x$ from $\mathscr{C}(\sigma(T))$ into $X$ is weakly compact).

Fact 2 [3, Theorem VI.7.6]. Let $\Omega$ be a compact Hausdorff space. Then every bounded linear operator from $\mathscr{C}(\Omega)$ into a weakly complete Banach space is weakly compact.

The following theorem shows that one can characterize the Banach spaces for which Berkson and Dowson's condition is sufficient for an operator to be scalar-type spectral as exactly those spaces which do not contain $c_{0}$.

Theorem 2. Suppose that $X$ is a complex Banach space. Then the following are equivalent:

(i) $X$ does not contain a subspace isomorphic to $c_{0}$;

(ii) every well-bounded operator on $X$ with a decomposition of the identity of bounded variation is real scalar-type spectral.

Proof. (i) $\Rightarrow$ (ii). this is an easy consequence of Theorem 1, Fact 1 and the fact that every map from the continuous functions on a compact Hausdorff space into a space not containing $c_{0}$ is weakly compact [5, Theorem 5]. 
(ii) $\Rightarrow$ (i). Suppose that $X$ contains a subspace $Y$ which is isomorphic to $c_{0}$. Then $Y$ is also isomorphic to the space $c$ of all convergent sequences. We shall follow the methods of [4] to embed a suitable operator on $c$ into $B(X)$. If we regard $Y$ as being identified with $c$, we can equip it with either of the two equivalent norms; $\|\cdot\|_{\infty}$ from $c$ and $\|\cdot\|_{X}$ from $X$. We shall assume that $K^{-1}\|\cdot\|_{X} \leq\|\cdot\|_{\infty} \leq K\|\cdot\|_{X}$.

Let $e_{n} \in Y$ be the element of $c$ which has 1 in the $n$th position and 0 elsewhere, and let $\varphi_{n}^{\prime}$ be the $n$th coordinate functional on $c$ (i.e., $\left\langle\sum \alpha_{k} e_{k}\right.$, $\left.\left.\varphi_{n}^{\prime}\right\rangle=\alpha_{n}\right)$. It is easy to check that $\left|\left\langle y, \varphi_{n}^{\prime}\right\rangle\right| \leq K\|y\|_{X}$ for all $y \in Y$. We shall denote by $\varphi_{n}$ a fixed norm-preserving extension of $\varphi_{n}^{\prime}$ to all of $X$.

Define $S$ on $X$ by $S x=\sum_{n=1}^{\infty}(1 / n)\left\langle x, \varphi_{n}\right\rangle e_{n}$. Note that for $x \in X$, $\left|\left\langle x, \varphi_{n}\right\rangle\right| \leq K\|x\|_{X}$, so that $(1 / n)\left\langle x, \varphi_{n}\right\rangle$ tends to zero as $n$ tends to $\infty$. Thus $S x \in Y$ for all $x \in X$. Now

$$
\begin{aligned}
\|S x\|_{X} & \leq K\|S x\|_{\infty} \\
& =K \sup _{n}\left|(1 / n)\left\langle x, \varphi_{n}\right\rangle\right| \\
& \leq K^{2} \sup _{n}(1 / n)\|x\|_{X}
\end{aligned}
$$

so $S$ is a bounded operator on $X$. Indeed, if $g$ is a polynomial then

$$
g(S) x=\sum_{n=1}^{\infty}\left(g\left(\frac{1}{n}\right)-g(0)\right)\left\langle x, \varphi_{n}\right\rangle e_{n}+g(0) x .
$$

Thus

$$
\begin{aligned}
\|g(S) x\| & \leq K \sup _{n}\left|\left(g\left(\frac{1}{n}\right)-g(0)\right)\left\langle x, \varphi_{n}\right\rangle\right|+\|g(0) x\|_{X} \\
& \leq\left(2 K^{2}+1\right) \sup _{[a, b]}|g|\|x\|_{X} .
\end{aligned}
$$

By Theorem 1 then, $S$ is a well-bounded operator which possesses a decomposition of the identity of bounded variation. However, as is shown in [2, Example 14.6], $S \mid Y$ is not a scalar-type spectral operator. Thus, by [2, Theorem 12.16], $S$ cannot be a scalar-type spectral operator on $X$.

\section{ACKNOWLEDGMENT}

The author would like to express his thanks to Dr. T. A. Gillespie for several helpful discussions regarding this paper.

\section{REFERENCES}

1. E. Berkson and H. R. Dowson, On uniquely decomposable well-bounded operators, Proc. London Math. Soc. (3) 22 (1971), 339-358.

2. H. R. Dowson, Spectral theory of linear operators, Academic Press, London, 1978.

3. N. Dunford and J. T. Schwartz, Linear operators, Part I: General theory, Wiley-Interscience, New York, 1958. 
4. T. A. Gillespie, Strongly closed bounded Boolean algebras of projections, Glasgow Math. J. 22 (1981), 73-75.

5. A. Pełczyński, Projections in certain Banach spaces, Studia Math. 19 (1960), 209-228.

Department of Mathematics, University of Edinburgh, James Clerk Maxwell Building, Mayfield RoAd, Edinburgh, United Kingdom, EH9 3JZ

Current address: School of Mathematics, Physics, Computing and Electronics, Macquarie University, N.S.W., 2109 Australia 